\title{
Playing with Fire: The Relationship between Mental Disorders and Prescription Opioid Abuse in a Rural Juvenile Court Sample
}

\author{
Keith F. Durkin, Ph.D. \\ Department of Psychology, Sociology and Criminal Justice \\ Ohio Northern University \\ 525 S Main St, Ada, OH 45810, USA \\ Wade A. Melton, LSW, PJJA, M.A.C.E \\ Hardin County Court of Common Pleas, Juvenile Division \\ Tristin M. Kilgallon, J.D., LL.M \\ Department of Psychology, Sociology and Criminal Justice \\ Ohio Northern University \\ 525 S Main St, Ada, OH 45810, USA
}

\begin{abstract}
The United States is in the ruthless grip of a terrible opioid epidemic that has hit rural areas especially hard. This horrible addiction often begins with the misuse of prescription opioids. This paper documents the relationship between co-occurring mental disorders and prescription opioid abuse in a sample of 99 youths involved with a juvenile court in a rural county in Ohio. Results indicate that juveniles who reported misusing prescription opioids were more likely than the other juveniles to report having any type of mental health problem, any type of externalizing problem, and any type of internalizing problem. Compared to other subjects, youths who reported opioid abuse were 2.5 times more likely to have symptoms consistent with a mood disorder (including depression) and more than 7 times more likely to report symptoms reflecting general anxiety disorder. Juveniles who reported misuse of prescription opioids were also more likely than their peers to report symptoms of ADD/ADHD and conduct disorder. Directions for future research and implications of the problem of prescription opioid abuse for juvenile courts are also considered.
\end{abstract}

Keywords: prescription opioid abuse; juvenile delinquency; mental health; opioid epidemic; comorbidity

\section{Introduction}

The epidemic of opioid abuse is a tremendous public health emergency in the United States, resulting in increasing numbers of deaths and emergency room visits from drug overdoses. Between 2000 and 2014 there was a $137 \%$ increase in drug poisoning deaths nationwide (Ruhm, 2017). Opioids were responsible for the vast majority of the approximately 64,000 overdose deaths in 2016 (Aliprantis\&Chen,2017). Drug overdoses are now the leading cause of accidental death in the United States, accounting for a greater number of fatalities than either suicides, traffic accidents, or gun violence (Ciccarone, 2017). However, unlike previous drug epidemics, the current crisis has hit rural America especially hard. Today the typical opioid addict is white and resides in a nonurban, often rural, area (Bowser, Fullilove, \& Word, 2017; Cicero, Ellis, Surratt, \& Kurtz, 2014). Compared to urban areas, rural areas have higher rates of opioid overdoses (Dunn et al., 2016) and opioid overdose deaths (Keyes, Cerda, Brady, Havens, \& Galea, 2014).

The abuse of prescription opioids is a major component of this crisis. The misuse of these drugs is a gateway or stepping-stone to eventual heroin use (Carlson, Nahhas, Martins, \& Daniulaityte, 2016). The vast majority of heroin users first abuse prescription opioids (Compton, Boyle, \& Wargo 2015; Maxwell, 2015), and individuals who abuse prescription opioids are several times more likely than their peers to turn to heroin use (Vosburg et al., 2016).Unfortunately, the misuse of prescription opioids by secondary school students nationally is on the rise, and it has almost doubled over the course of the last decade (Godley et al., 2017). 
Aside from the well-documented link to the eventual initiation of heroin use, there are other aspects of prescription opioid abuse among adolescents that are troubling (see Sharma, Bruner, Barnett, \& Fishman, 2016). For example, an overdose does not require an addiction. It can occur the very first time someone experiments with abusing prescription opioids. Unfortunately, research indicates that rural youths are at an even greater risk of engaging in prescription opioid abuse than their suburban and urban peers (McHugh, Nielsen, \& Weiss, 2015; Monnat \& Rigg, 2015; Palmar, Shearston, Dawson, Mateu-Gelabert, \& Ompad, 2016; Park, Melander, \& Sanchez,2016).This problem is further complicated by the fact that rural areas have a substantially lower availability of opioid treatment compared to more populated locations (Dunn et al., 2016; Hall et al., 2008).

Overall, children and adolescents who abuse substances are more likely than their peers to have mental health problems (Chan, Dennis, \& Funk, 2008). Previous research has identified a number of specific psychological disorders that are linked to substance abuse among adolescents. These conditions include: ADD/ADHD (Lansford et al., 2008; Zulauf, Sprich, Safren, \& Wilens, 2014); conduct disorder (Conway, Swendsen, Husky, He, \& Merikangas, 2016; Godley, Smith, Passetti, \&S ubramaniam, 2014); depression (Ayres, Pontes, \& Pontes, 2017; Darzdowski, Jaggi, Borre, \& Kliewer, 2015); traumatic stress disorder (Drazdowski et al., 2015; Godley et al., 2014); and general anxiety disorder (Conway et al., 2016).

Although there has not been a great deal of research focusing specifically on the relationship between adolescent opioid abuse and mental health problems, the limited body of literature on the subject suggests that the two are related. For instance, Godley et al. (2017) found that young people who abuse prescription opioids generally have a greater degree of mental health problems than youths who abuse other substances, such as marijuana. Moreover, according to Monnat and Rigg (2015), the misuse of prescription opioids by juveniles is positively associated with experiencing a major depressive episode, as well as having a history of hospitalization for emotional problems. Furthermore, research indicates that anxiety disorders are also positively related to opioid abuse by juveniles (Welsh et al., 2017). Finally, Edlund et al. (2015) found prescription opioid abuse is two times more likely among adolescents with a history of an episode of major depression.

One concern is that mental health issues may be driving prescription opioid abuse among young people. A common explanation for the co-occurrence between mental health problems and substance abuse is that the drug abuser turns to substances as a means of "self-medication" to alleviate the distressing psychological symptoms of a mental disorder (Edlund et al., 2015). In turn, these mental disorders complicate the treatment of substance abuse and those individuals with co-occurring mental health problems have worse substance abuse treatment outcomes than their peers (Godley et al., 2014; Schuler, Vasilenko, \& Lanza, 2015). Co-occurring substance abuse problems and mental disorders also carry a greater disease burden than either condition individually, including both suicidal behavior and criminal justice system involvement (Fink et al., 2015). Consequently, the problem of these co-occurring mental disorders among juveniles who abuse prescription opioids are frequently a major issue for juvenile courts. Accordingly, juvenile courts have to address the complex set of issues these specific juveniles present. This troublesome situation is further complicated in rural areas due to the lack of available resources for substance abuse and mental health treatment (Dunn et al., 2016; Hall et al., 2008; Monnat \& Rigg, 2015).

The purpose of this research was to examine the relationship between mental health problems and prescription opioid abuse in a sample of subjects from a juvenile court in a rural area of Ohio that has a serious problem with heroin addiction in the adult population. There were three research hypothesis in this study. First, juveniles who reported prescription opioid abuse would be more likely to report mental health problems than juveniles who did not misuse prescription opioids. Second, juveniles who reported prescription opioid abuse would be more likely than their peers to report symptoms consistent with an externalizing disorder (i.e., ADD/ADHD and conduct disorder). Finally, juveniles with a history of opioid abuse would be more likely than their peers to report mental health problems consistent with an internalizing disorder (i.e., mood disorder, general anxiety disorder, and traumatic stress disorder).

\section{Methods}

\section{Sample}

The subjects were 99 juveniles who were participating in a rural Ohio juvenile court due to their involvement in criminal and/or delinquent behavior. 
The majority of subjects $(61 \%)$ were male. In terms of race, $87 \%$ were white, $9 \%$ were bi-racial, and the remaining $4 \%$ represented other racial groups. The mean age of the sample was 15.4 years old.

\section{Instrument}

The Global Appraisal of Individual Needs (GAIN) (see Ives, Funk, Ihnes, Feeney, \& Dennis, 2012) was administered to all of these juveniles as part of the regular court intake procedures. The GAIN is a standardized comprehensive biopsychosocial battery of assessment measures designed to combine both clinical decisions and research into a single interview (Titus, Dennis, Lennox, \& Scott, 2008). It is used to support diagnosis, placement, treatment planning, and service utilization. The GAIN has eight core sections including mental health and substance abuse. These items are combined into more than 100 indexes, scales, and subscales. The GAIN scales have been constructed using Rasch measurement analysis and they have been normed with over 100,000 subjects (Scott, Dennis, \& Lurigio, 2015). The primary mental health scales have an outstanding degree of internal consistency (Alpha of .9 or greater) and subscales have a very strong level of internal consistency (Alpha of .7 or higher) (Chan et al., 2008; Titus et al., 2008). The diagnostic impressions produced by the GAIN are strongly correlated with blind psychiatric diagnoses, collateral reports, and treatment records (Ives et al., 2012). The GAIN provides a count of various symptoms experienced by a respondent over the course of the last twelve months. These symptoms are consistent with a variety of common psychological disorders as reflected by the respective DSM-IV criteria.

\section{Measures}

The presence of an internalizing disorder was indicated by a respondent reporting a substantial number of symptoms that are consistent with a major internalizing problem (i.e., anxiety, mood disorder, suicidal ideation/behavior, and trauma). Past year general anxiety disorder was characterized by reporting at least three general symptoms plus two required symptoms. For past year mood disorder (including depression), the endorsement of five or more symptoms, including three compulsory ones, was required. Past year traumatic stress disorder was characterized by the endorsement of at least five symptoms. The existence of an externalizing disorder was categorized by a respondent reporting a substantial number of symptoms consistent with a major externalizing problem (i.e., ADHD/ADD and conduct disorder). Past year ADHD/ADD was classified by the endorsement of a minimum of six symptoms related to hyperactivity/impulsivity type, inattention type, or both. For past year conduct disorder, the endorsement of at least three symptoms was required. Lifetime prescription opioid abuse was measured by asking subjects to self-report whether or not they had ever used prescription opioids in any situation that was not under the direction of a medical doctor.

\section{Data Analysis}

The data from the GAIN were entered electronically in a data file that was then transferred into SPSS (Statistical Package for the Social Sciences) format. The Chi-Square Test of Independence (Sheskin, 2003) was utilized to determine if there were statistically significant differences in the prevalence of the respective mental health problems between juveniles who had abused prescription opioids and those who had not.

\section{Results}

The relationship between specific mental health problems and prescription opioid abuse among subjects in this sample is shown in Table 1. Overall, twenty-one percent of the sample reported engaging in this behavior. Those youths who abused prescription opioids were significantly more likely than the other youths to report having at least one mental health problem. In fact, all of those youths who reported misusing prescription opioids reported symptoms of at least one mental health problem.

The juveniles with a history of prescription opioid misuse were significantly more likely than their peers to report symptoms of an externalizing disorder (95.2\% vs. 51.9\%). ADD/ADHD problems were significantly more prevalent among youths who reported prescription opioid abuse $(61.9 \%)$ than those who didn't report this behavior $(34.2 \%)$. Subjects with a history of prescription opioid misuse were almost twice as likely as their peers to indicate symptomology that is consistent with a conduct disorder. Those juveniles who report that they had abused prescription opioids were nearly twice as likely as their peers to have problems related to an internalizing disorder. 
Compared to their peers, the opioid abusers were 2.5 times more likely to report symptoms consistent with a mood disorder (including depression), and more than 7 times more likely to report symptoms reflecting general anxiety disorder. However, no significant difference was found between the two groups in terms of traumatic stress disorder symptoms. Finally, we considered situations where subjects reported symptoms of co-occurring internalizing/externalizing disorders. Juveniles who abused prescription opioids were more than two times more likely than their peers to report co-occurring internalizing/externalizing symptoms (61.9\% vs. $23.4 \%)$.

\section{Table 1. Relationship between Specific Mental Health Problems and Prescription Opioid Abuse} History of Prescription Opioid Abuse

\begin{tabular}{|c|c|c|c|}
\hline & $\begin{array}{c}\text { Yes } \\
(n=21)\end{array}$ & $\begin{array}{c}\text { No } \\
(n=78)\end{array}$ & $\mathbf{X}^{2}$ \\
\hline \multicolumn{4}{|c|}{ Any Mental Health Problem } \\
\hline Yes & $100.0 \%$ & $61.8 \%$ & \\
\hline No & $0.0 \%$ & $38.2 \%$ & $11.43 * *$ \\
\hline \multicolumn{4}{|c|}{ Any Externalizing Disorder } \\
\hline Yes & $95.2 \%$ & $51.9 \%$ & \\
\hline No & $4.8 \%$ & $48.1 \%$ & $13.03 * *$ \\
\hline \multicolumn{4}{|l|}{ ADD/ADHD } \\
\hline Yes & $61.9 \%$ & $34.2 \%$ & \\
\hline No & $38.1 \%$ & $65.8 \%$ & $5.25^{*}$ \\
\hline \multicolumn{4}{|c|}{ Conduct Disorder } \\
\hline Yes & $76.2 \%$ & $40.3 \%$ & \\
\hline No & $23.8 \%$ & $59.7 \%$ & $8.54 * *$ \\
\hline \multicolumn{4}{|c|}{ Internalizing Disorder } \\
\hline Yes & $66.7 \%$ & $35.1 \%$ & \\
\hline No & $33.3 \%$ & $64.9 \%$ & $6.77 * *$ \\
\hline \multicolumn{4}{|c|}{ Mood Disorder (Including Depression) } \\
\hline Yes & $61.9 \%$ & $24.7 \%$ & \\
\hline No & $38.1 \%$ & $75.3 \%$ & $10.40 * *$ \\
\hline \multicolumn{4}{|c|}{ General Anxiety Disorder } \\
\hline Yes & $28.6 \%$ & $3.9 \%$ & \\
\hline No & $71.4 \%$ & $96.1 \%$ & $12.05 * *$ \\
\hline \multicolumn{4}{|c|}{ Traumatic Stress Disorder } \\
\hline Yes & $42.9 \%$ & $22.1 \%$ & \\
\hline No & $57.1 \%$ & $77.9 \%$ & 3.66 \\
\hline \multicolumn{4}{|c|}{ Co-Occurring Internalizing/Externalizing Disorders } \\
\hline Yes & $61.9 \%$ & $23.4 \%$ & \\
\hline No & $38.1 \%$ & $76.6 \%$ & $11.43 * *$ \\
\hline
\end{tabular}

\section{Discussion}

The aim of the current study was to examine the relationship between mental health problems and prescription opioid abuse in a sample of subjects from a rural juvenile court. As hypothesized, youths with a history of prescription opioid abuse were more likely than other youths to have mental health problems. With only one exception (i.e., traumatic stress disorder), juveniles who abused prescription opioids were more likely than their peers to experience symptoms of a variety of specific mental disorders (i.e., ADD/ADHD, conduct disorder, mood disorder including depression, and general anxiety disorder). Additionally, subjects reporting prescription opioid misuse were more likely to present a greater degree of clinical severity in terms of mental health problems (i.e., co-occurring internalizing/ externalizing disorders) than juveniles who did not report engaging in that behavior. 
Such findings are consistent with previous research that indicates that adolescents who abuse prescription opioids are more likely than their peers to have mental health problems (Godley et al., 2017), as well as to have specific mental health problems including depression (Edlund et al., 2015; Monnat \& Rigg, 2015) and anxiety disorders (Welsh et al., 2017).

An important implication of this research is that opioid abuse and mental health problems should not be considered separate issues because the two variables co-occur. For juvenile courts, effective intake assessment involves simultaneously screening for both substance abuse and mental health problems.

For substance abuse treatment to be successful, co-occurring psychological problems must also be addressed (Goldner, Lusted, Roerecke, Rehm, \& Fischer, 2014). Consequently, an integrated approach that simultaneously deals with those co-occurring substance and mental health issues is strongly recommended (Godley et al., 2014; Lansford et al., 2008; Schuler et al., 2015). Failure to treat the co-occurring mental health issues is setting the substance abuser up for failure. Once the distressing symptoms of the mental health problem that the person was trying to avoid reoccur, it can pressure them to relapse (Meyer, McWey, McKendrick, \& Henderson, 2010). However, it is important to note that for juvenile courts in rural locations, access to substance abuse treatment and psychological services may be very limited (Dunn et al., 2016; Hall et al., 2008; Monnat \& Rigg, 2015). Consequently, court teams must be creative and resourceful to see that youths are getting the needed services.

This phenomenon also has important implications for juvenile probation practice. Many young people who abuse prescription opioids become involved with the juvenile court and are placed on probation. Juvenile Probation Officers (JPO) are charged with the vital task of supervising and monitoring young offenders to ensure their compliance with the court mandated conditions of their probation. Because of the authority granted to JPOs by the courts, and their close relationship with support services in the community, they should be among the vanguard in any attempt to address the juvenile opioid abuse crisis. Unlike other street drugs, prescription opioids are often readily available without having to visit a drug dealer (Sharma et al., 2016). Juveniles can often find these drugs in medicine cabinets. Nonetheless, tools available to the JPO that may assist them in identifying probationers who abuse opioids. First, regular home visits and maintaining open lines of communication with family members may alert the JPO to the presence of prescription opioids in the home. While the mere presence of the drugs in the home is not, in and of itself reason for alarm, it should be noted that if not securely stored, prescription drugs may be diverted for illicit use. Second, the authority to require the probationer submit to random drug screenings is vital in identifying prescription opioid abuse issues. A positive test result can lead the JPO take curative action to remedy the problem, or if necessary, punitive action. Finally, JPOs should be aware of the mental health issues a probationer is facing and be sensitive to any changes in their condition or failure to comply with treatment expectations.

When it comes to addressing the misuse of prescription opioids by young people, the stakes are incredibly high. Confronting this issue during the childhood and adolescent years is the key to preventing future heroin use its devastating costs to the individual and society. Even the so-called "experimental" misuse of prescription opioids puts young people at risk for a fatal overdose. An especially concerning new development involves counterfeit prescription opioid tablets that have been adulterated with the deadly synthetic opioid fentanyl being sold by drug dealers (Sutter et al., 2017; Vo, van Wijk, Lynch, Wu, \& Smollin, 2016). Unsuspecting juveniles could easily become the victims of fatal overdoses if they access these counterfeit pills. Efforts aimed at preventing prescription opioid misuse before it starts are essential. Because of the apparent strong association between mental health problems and prescription opioid abuse, efforts to treat the mental health issues of young people before they start abusing drugs are especially important (Lansford et al., 2008; Schuler et al., 2015).

\section{Limitations}

There are several limitations associated with this study that should be acknowledged. First, the data were collected from a single juvenile court in a rural Ohio county. Therefore, the generalizability of these findings is unknown. Second, GAIN data on mental health problems are not diagnoses, but rather a measure of the extent to which various diagnostic criteria are or are not present (see Chan et al., 2008). However, the diagnostic impressions produced by the GAIN are strongly correlated with blind psychiatric diagnoses, treatment records, and collateral reports (Ives et al., 2012).Third, a cross-sectional research design was used so we are unable to establish any sort of causal relationship between mental disorders and prescription opioid use. 
There is a larger debate regarding the causality of these variables, specifically whether mental illness precedes drug use, drug use precedes mental illness, or both have a "common cause" (see Fischer, Murphy, Kurdyak, \& Goldner, 2016). Obviously, the current undertaking contributes nothing to that larger debate. However, for researchers and professionals working with courts and treatment agencies that are on the front lines of the current opioid epidemic, addressing such "chicken or egg" academic questions aren't an immediate priority at this trying time.

\section{Future Directions}

The research reported in this paper was exploratory in nature and was limited to a single juvenile court. Future research that utilizes much a much larger and more diverse sample of young people would be particularly useful in establishing the specific relationships between juvenile opioid abuse and mental health problems.

One way to accomplish this would be to pool the GAIN data from multiple juvenile court programs. Another future objective for research would be to investigate the relationship between specific mental health problems and treatment outcomes for juvenile prescription opioid abusers. The identification of specific disorders linked to specific outcomes would be extremely valuable in treatment planning. Finally, an eventual goal would be longitudinal studies that can determine the specific ordering of mental health variables and substance abuse.

\section{Conclusion}

The current epidemic of opioid abuse in the United States constitutes an unprecedented public health emergency. This epidemic has hit rural areas especially hard. Unfortunately, rural areas are generally ill-equipped to provide opioid abusers with the needed rehabilitation services. The vast majority of heroin addicts first abuse prescription opioids and this prescription opioid abuse is often initiated during adolescence. The current research documented a very strong association between mental health problems and prescription opioid misuse in a sample of young people involved with a rural Ohio juvenile court. These findings suggest that youths who are referred to the juvenile court should be simultaneously screened for mental health problems and substance abuse at intake. Moreover, effective treatment must address both sets of issues. Dealing with these co-occurring issues when substance abusers are still relatively young and yet to initiate heroin use seems to be a logical step in preventing the continuation of this deadly epidemic. Because these young people often end up involved in the juvenile justice system, juvenile courts can play an integral role in combating the nationwide opioid epidemic.

\section{Acknowledgements}

This research was supported by an Ohio Department of Youth Services Competitive RECLAIM Grant.

\section{References}

Aliprantis, D., \& Chen, A. (2017). The opioid epidemic and the labor market. Economic Commentary 2017(15), $1-5$.

Ayres, C. G., Pontes, N. M., \& Pontes, M. C. (2017). Understanding the nonmedical use of prescription medications in the US high school adolescents. The Journal of School Nursing, 33(4), 269-276.

Bowser, B., Fullilove, R., \& Word, C. (2017). Is the new heroin epidemic really new? Racializing heroin. Journal of the National Medical Association, 109(1), 28-32.

Carlson, R. G., Nahhas, R. W., Martins, S. S., \& Daniulaityte, R. (2016). Predictors of transition to heroin use among initially non-opioid dependent illicit pharmaceutical opioid users: A natural history study. Drug \& Alcohol Dependence, 160, 127-134.

Chan, Y. F., Dennis, M. L., \& Funk, R. R. (2008). Prevalence and comorbidity of major internalizing and externalizing problems among adolescents and adults presenting to substance abuse treatment. Journal of Substance Abuse Treatment, 34(1), 14-24.

Ciccarone, D. (2017). Fentanyl in the US heroin supply: A rapidly changing risk environment. International Journal of Drug Policy, 46, 107-111.

Cicero, T. J., Ellis, M. S., Surratt, H. L., \& Kurtz, S. P. (2014). The changing face of heroin use in the United States: A retrospective analysis of the past 50 years. JAMA Psychiatry, 71(7), 821-826.

Compton, W. M., Boyle, M., \& Wargo, E. (2015). Prescription opioid abuse: Problems and responses. Preventive Medicine, 80, 5-9. 
Conway, K. P., Swendsen, J., Husky, M. M., He, J. P., \& Merikangas, K. R. (2016). Association of lifetime mental disorders and subsequent alcohol and illicit drug use: results from the National Comorbidity Survey-Adolescent Supplement. Journal of the American Academy of Child \& Adolescent Psychiatry, 55(4), 280-288.

Drazdowski, T. K., Jäggi, L., Borre, A., \&Kliewer, W. L. (2015). Use of prescription drugs and future delinquency among adolescent offenders. Journal of Substance Abuse Treatment, 48(1), 28-36.

Dunn, K.E., Barrett, F.S., Yepez-Laubach, C., Meyer, A.C., Hruska, B.J., Petrush, K., Berman, S., Sigmon, S.C., Fingerhood, M. \&Bigelow, G.E. (2016). Opioid overdose experience, risk behaviors, and knowledge in drug users from a rural versus an urban setting. Journal of Substance Abuse Treatment, 71, 1-7.

Edlund, M. J., Forman-Hoffman, V. L., Winder, C. R., Heller, D. C., Kroutil, L. A., Lipari, R. N., \&Colpe, L. J. (2015). Opioid abuse and depression in adolescents: results from the National Survey on Drug Use and Health. Drug \& Alcohol Dependence, 152, 131-138.

Fink, D. S., Hu, R., Cerdá, M., Keyes, K. M., Marshall, B. D., Galea, S., \& Martins, S. S. (2015). Patterns of major depression and nonmedical use of prescription opioids in the United States. Drug \& Alcohol Dependence, 153, 258-264.

Fischer, B., Murphy, Y., Kurdyak, P., \& Goldner, E. M. (2016). Depression-A major but neglected consequence contributing to the health toll from prescription opioids? Psychiatry Research, 243, 331-334.

Godley, M. D., Passetti, L. L., Subramaniam, G. A., Funk, R. R., Smith, J. E., \& Meyers, R. J. (2017). Adolescent community reinforcement approach implementation and treatment outcomes for youth with opioid problem use. Drug and Alcohol Dependence, 174, 9-16.

Godley, S. H., Smith, J. E., Passetti, L. L., \& Subramaniam, G. (2014). The Adolescent Community Reinforcement Approach (A-CRA) as a model paradigm for the management of adolescents with substance use disorders and co-occurring psychiatric disorders. Substance Abuse, 35(4), 352-363.

Goldner, E. M., Lusted, A., Roerecke, M., Rehm, J., \& Fischer, B. (2014). Prevalence of Axis-1 psychiatric (with focus on depression and anxiety) disorder and symptomatology among non-medical prescription opioid users in substance use treatment: systematic review and meta-analyses. Addictive Behaviors, 39(3), 520531.

Hall, J. A., Smith, D. C., Easton, S. D., An, H., Williams, J. K., Godley, S. H., \& Jang, M. (2008). Substance abuse treatment with rural adolescents: Issues and outcomes. Journal of Psychoactive Drugs, 40(1), 109120.

Ives, M., Funk, R., Ihnes, P., Feeney, T. \& Dennis, M. (2012). Global Appraisal of Individual Needs evaluation manual. Chicago, IL: Chestnut Health Systems.

Keyes, K. M., Cerdá, M., Brady, J. E., Havens, J. R., \& Galea, S. (2014). Understanding the rural-urban differences in nonmedical prescription opioid use and abuse in the United States. American Journal of Public Health, 104(2), e52-e59.

Lansford, J. E., Erath, S., Yu, T., Pettit, G. S., Dodge, K. A., \& Bates, J. E. (2008). The developmental course of illicit substance use from age 12 to 22: Links with depressive, anxiety, and behavior disorders at age 18 . Journal of Child Psychology and Psychiatry, 49(8), 877-885.

Maxwell, J. C. (2015). The pain reliever and heroin epidemic in the United States: Shifting winds in the perfect storm. Journal of Addictive Diseases, 34(2-3), 127-140.

McHugh, R. K., Nielsen, S., \& Weiss, R. D. (2015). Prescription drug abuse: from epidemiology to public policy. Journal of Substance Abuse Treatment, 48(1), 1-7.

Meyer, A. S., McWey, L. M., McKendrick, W., \& Henderson, T. L. (2010). Substance using parents, foster care, and termination of parental rights: The importance of risk factors for legal outcomes. Children and Youth Services Review, 32(5), 639-649.

Monnat, S. M., \&Rigg, K. K. (2015). Examining rural/urban differences in prescription opioid misuse among US adolescents. The Journal of Rural Health, 32(2), 204-218.

Palamar, J. J., Shearston, J. A., Dawson, E. W., Mateu-Gelabert, P., \& Ompad, D. C. (2016). Nonmedical opioid use and heroin use in a nationally representative sample of us high school seniors. Drug \& Alcohol Dependence, 158, 132-138.

Park, N. K., Melander, L., \& Sanchez, S. (2016). Nonmedical prescription drug use among Midwestern rural adolescents. Journal of Child \& Adolescent Substance Abuse, 25(4), 360-369. 
Ruhm, C. J. (2017). Geographic variation in opioid and heroin involved drug poisoning mortality rates. American Journal of Preventive Medicine, 53(6), 745-753.

Schuler, M. S., Vasilenko, S. A., \& Lanza, S. T. (2015). Age-varying associations between substance use behaviors and depressive symptoms during adolescence and young adulthood. Drug \& Alcohol Dependence, $157,75-82$.

Scott, C. K., Dennis, M. L., \& Lurigio, A. J. (2015). Comorbidity among female detainees in drug treatment: An exploration of internalizing and externalizing disorders. Psychiatric Rehabilitation Journal, 38(1), 35-44.

Sharma, B., Bruner, A., Barnett, G., \& Fishman, M. (2016). Opioid use disorders. Child and Adolescent Psychiatric Clinics, 25(3), 473-487.

Sheskin, D. J. (2003). Handbook of parametric and nonparametric statistical procedures. New York, NY: CRC Press.

Sutter, M.E., Gerona, R.R., Davis, M.T., Roche, B.M., Colby, D.K., Chenoweth, J.A., Adams, A.J., Owen, K.P., Ford, J.B., Black, H.B. \& Albertson, T.E. (2017). Fatal fentanyl: One pill can kill. Academic Emergency Medicine, 24(1), 106-113.

Titus, J. C., Dennis, M. L., Lennox, R., \& Scott, C. K. (2008). Development and validation of short versions of the internal mental distress and behavior complexity scales in the Global Appraisal of Individual Needs (GAIN). The Journal of Behavioral Health Services \& Research, 35(2), 195-214.

Vo, K.T., van Wijk, X.M.R., Lynch, K.L., Wu, A.H.B., \& Smollin, C.G. (2016). Counterfeit Norco poisoning outbreak-San Francisco Bay Area, California, March 25-April 5, 2016. MMWR. Morbidity and Mortality Weekly Report, 65, 420-423.

Vosburg, S. K., Eaton, T. A., Sokolowska, M., Osgood, E. D., Ashworth, J. B., Trudeau, J. J, Muffett-Lipinski, M. \& Katz, N. P. (2016). Prescription opioid abuse, prescription opioid addiction, and heroin abuse among adolescents in a recovery high school: A pilot study. Journal of Child \& Adolescent Substance Abuse, 25(2), 105-112.

Welsh, J. W., Knight, J. R., Hou, S.S.Y., Malowney, M., Schram, P., Sherritt, L., \& Boyd, J. W. (2017). Association between substance use diagnoses and psychiatric disorders in an adolescent and young adult clinic-based population. Journal of Adolescent Health, 60(6), 648-652.

Zulauf, C. A., Sprich, S. E., Safren, S. A., \& Wilens, T. E. (2014). The complicated relationship between attention deficit/hyperactivity disorder and substance use disorders. Current psychiatry reports, 16(3), 436-447. 